\title{
Hematologic Features of Children and Adolescent Patients with Acute Hypersensitivity Reactions on Drugs and Food
}

\author{
Tatyana I. Eliseeva $\mathbb{D}^{1},{ }^{1}$ Ivan I. Balabolkin, ${ }^{2}$ Natalia A. Geppe, ${ }^{3}$ Elena V. Tush $\mathbb{D}^{1}{ }^{1}$ \\ Dmitry Yu. Ovsyannikov $\mathbb{D}^{4},{ }^{4}$ Nadezhda S. Podchernyaeva, ${ }^{3}$ Polina V. Trushanina $\mathbb{D},{ }^{1,5}$ \\ Stanislav K. Ignatov $\mathbb{D}$, ${ }^{6}$ Nailya I. Kubysheva $\mathbb{D}^{7}{ }^{7}$ Vilya A. Bulgakova $\mathbb{D}^{2,3,8}$ \\ and Olga V. Khaletskaya ${ }^{1}$ \\ ${ }^{1}$ Federal State Budgetary Educational Institution of Higher Education "Privolzhsky Research Medical University" \\ of the Ministry of Health of the Russian Federation, Minin and Pozharsky Square 10/1, Nizhny Novgorod 603005, Russia \\ ${ }^{2}$ Federal State Autonomous Institution "National Medical Research Center of Children's Health" of the \\ Ministry of Health of the Russian Federation, Lomonosovsky Prospekt 2, Build. 1, Moscow 119991, Russia \\ ${ }^{3}$ Federal State Autonomous Educational Institution of Higher Education I.M. Sechenov First Moscow \\ State Medical University of the Ministry of Health of the Russian Federation (Sechenov University), Trubetskaya St. 8/2, \\ Moscow 119991, Russia \\ ${ }^{4}$ Department of Children's Diseases, Peoples' Friendship University of Russia (RUDN University), 6 Miklukho-Maklaya St., \\ Moscow 117198, Russia \\ ${ }^{5}$ Pediatrician Children's City Clinical Hospital No. 1, 1st Children's City Clinical Hospital of Nizhny Novgorod, \\ Gagarin Avenue 76, Nizhny Novgorod 60308, Russia \\ ${ }^{6}$ Lobachevsky State University of Nizhny Novgorod, Gagarina Avenue 23, Nizhny Novgorod 603950, Russia \\ ${ }^{7}$ Kazan Federal University, 18 Kremlyovskaya St., Kazan 420008, Russia \\ ${ }^{8}$ N. I. Pirogov Russian National Research Medical University, Ostrovitianov St. 1, Moscow 117997, Russia
}

Correspondence should be addressed to Nailya I. Kubysheva; aibolit70@mail.ru

Received 28 September 2019; Accepted 23 January 2020; Published 27 February 2020

Academic Editor: Enrico Heffler

Copyright (C) 2020 Tatyana I. Eliseeva et al. This is an open access article distributed under the Creative Commons Attribution License, which permits unrestricted use, distribution, and reproduction in any medium, provided the original work is properly cited.

Hematological parameters and blood biochemical markers were measured in 131 children and adolescent patients (70 boys) aged 2 to 17 years with acute hypersensitivity reactions induced by food (59 patients) and medicines ( 72 patients) in order to establish differences in clinical manifestations and hematological parameters in children with food and drug hypersensitivity and to elaborate the hematological criteria for differentiating the possible pathophysiological mechanisms of various types of hypersensitivity. Both groups of patients had comparable clinical symptoms with a predominance of skin lesions. The significant differences between the groups with drug- and food-induced hypersensitivity reactions were found in their red blood characteristics. In patients with hypersensitive reactions to drugs, significantly lower levels of erythrocytes and hemoglobin were found, while the median values of these parameters did not exceed the limits of reference values. These differences persisted also in the analysis of hemoglobin values, analyzed with accounting for the age and sex of patients. The reduction of hemoglobin was not accompanied by an increase in bilirubin in these patients. Thus, this fact does not support the assumption about the drug-induced hemolysis as a main effect influencing the hematological parameters. Hemogram evaluation performed during 7-10 days after admission demonstrated a higher level of hemoglobin in both groups. The biochemical markers were not significantly distinguished except bilirubin and alkaline phosphatase which were higher in patients with food-induced hypersensitivity. 


\section{Introduction}

Hypersensitivity reactions (HRs) are immune responses that are exaggerated or inappropriate against an antigen or allergen. HRs are very common: $15 \%$ of the world population are affected by any type of allergic reaction during their lives $[1,2]$. In the second half of the twentieth century, allergic diseases have become more abundant. Although most of them are self-limiting, they may adversely affect the quality of life and sometimes become life-threatening as well [3].

Of great interest are the differences in the mechanism of the occurrence of food hypersensitivity reactions (FHRs) and drug hypersensitivity reactions (DHRs), which often have phenotypically similar clinical manifestations $[4,5]$. The most frequent clinical manifestations of FHR and DHR are heterogeneous skin symptoms, although there may be involvement of other organs and systems, including the development of anaphylaxis. Nevertheless, there is marked heterogeneity of clinical presentations for FHR and DHR, which poses a challenge to successful management and treatment.

On the contrary, proinflammatory cytokines play a great role in the development of drug and food hypersensitivity reactions, as well as anemia of chronic disease [6-8]. So, theoretically, we found the hematological features in the patients with hypersensitivity reactions.

Recent publications by G. Jordakieva and coauthors revealed that one of the obscure phenomena, when a causally significant allergen is introduced into a sensitized macroorganism, is a decrease in hemoglobin and erythrocytes $[9,10]$. However, we failed to find any reports on the characteristic hematological features in the patients with hypersensitivity reactions in clinically manifested syndromes of skin damages and/or anaphylaxis. There are also no studies on comparison of hematological characteristics during the hypersensitivity reactions induced by different factors, e.g., during the DHR and food allergy. We found a single study by Sang-Yoon Kim and coauthors, including the analysis of some hematological parameters and their comparison with drug-induced anaphylaxis and in patients with anaphylaxis caused by food. However, in this study, a comparison was made between the total content of leukocytes and eosinophils in the peripheral blood and some biochemical parameters, but no comparison of red blood cell indices was presented. [11].

The elucidation of these differences could answer the question on the similarity or difference in the pathological mechanisms of food- and drug-induced hypersensitivity and point out the clinical and laboratory markers making possible differential diagnostics of these similar syndromes. In the present study, we consider these hematological features in the patients with the acute hypersensitivity reactions induced by food and drugs in children and adolescent patients. The main goal of this study is to determine the most significant differences in the blood cell characteristics and various blood biochemical parameters during the food and drug hypersensitivity reactions with a similar clinical picture.

\section{Materials and Methods}

2.1. Regulations and Criteria. A retrospective observational study was carried out using the medical histories of patients treated during 2016-2017 for acute allergic conditions in the 1st Children's City Clinical Hospital, Nizhny Novgorod, Russia. The study was conducted in accordance with the Helsinki Declaration, adopted in June 1964 (Helsinki, Finland), and revised in October 2000 (Edinburgh, Scotland). It was approved by the local Research Ethics Committee of Privolzhsky Research Medical University, Nizhny Novgorod, Russia, Protocol No. 13 of 10.10.16. In this research, we should not obtain the waived informed consent of patients because it was a retrospective chart review study. However, patient anonymity was preserved using the methods approved by the Ethics Committee.

2.2. Inclusion Criteria. Inclusion criteria include medical history with clinical manifestations of acute reactions of hypersensitivity, associated with drugs, or medical history with food intake (hypersensitivity to food) in the absence of the use of any drugs for the previous 4 weeks. Criteria for exclusion were the presence of symptoms of acute infection and fever.

2.3. Characteristics of Patients. A total of 131 children aged 2 to 17 years (mean age $8.22(7.54 ; 8.91)$ years; boys $53.4 \%$ (70/131), girls 46.6\% (61/131)) were studied. Among them, 59 patients included in the food hypersensitivity group (FH group) were treated for a severe allergic reaction after food provocation, and 72 patients were on treatment for the acute allergic reactions associated with drug hypersensitivity, drug hypersensitivity group (DH group).

History of allergic disease including food hypersensitivity, drug hypersensitivity, atopic dermatitis (AD), urticaria (URT), allergic rhinitis, and asthma was present in most patients $(70.2 \%$; 92/131), with comparable proportion of patients who had a history of allergic disease by FHR (62.7\%; 37/59) and $\operatorname{DHR}(76.4 \% ; 55 / 72), p=0.14$.

In the previous history, the hypersensitivity reactions to food among all the patients were observed in $42.7 \%(56 / 131)$ of the children, in the $\mathrm{FH}$ group in $35.6 \%$ (21/59) of the patients, and in the DH group in $48.6 \%$ (35/72) of the patients, $p=0.19$; hypersensitive reactions to medications in the history as a whole were observed in $24.4 \%(32 / 131)$ of the children, in the FH group in $11.9 \%(7 / 59)$, which is less than those in the DH group (34.7\% (25/72) of the patients), $p=0.006$.

The presence in the history of hypersensitivity reactions to both food and drug allergens was recorded only in the $\mathrm{DH}$ group, and the proportion of such patients was $19.4 \%$ $(14 / 72)$.

There were no statistically significant differences in the incidence of $\mathrm{AD}, \mathrm{URT}, \mathrm{AR}$, and/or DA between the FH and $\mathrm{DH}$ groups; in general, the occurrence of these pathological conditions was $25.9 \%$ (34/131) for BP, $23.7 \%$ (31/131) for URT, and $9.2 \%(12 / 131)$ for AR and/or BA. 
TABLE 1: Clinical characteristics of the patients. The data are presented as group fraction (\%) and the absolute values (in parentheses). The $95 \%$ confidence limits for the sampled values are given in brackets.

\begin{tabular}{|c|c|c|c|}
\hline & \multicolumn{2}{|c|}{ Group of patients } & \multirow{2}{*}{ Statistics $^{\mathrm{a}}$} \\
\hline & FH group & DH group & \\
\hline Number of patients & 59 & 72 & \\
\hline Boys & 37 & 33 & $\chi^{2}=3.71, p=0.054$ \\
\hline Girls & 22 & 39 & $\chi=3.1, p=0.054$ \\
\hline Age (years) & 8.31 [7.34; 9.27] & $8.18[7.17 ; 9.18]$ & $\begin{array}{c}t=0.18, p=0.86 \\
W=2038.5, p=0.7\end{array}$ \\
\hline Age (month) & $106.0[94.27 ; 117.7]$ & $103.2[91.1 ; 115.2]$ & $\begin{aligned} t & =0.33, p=0.74 \\
W & =2015.0, p=0.62\end{aligned}$ \\
\hline \multicolumn{4}{|l|}{ Prescribed therapy } \\
\hline Glucocorticosteroids dose (mg/kg per day) & $2.18[1.90 ; 2.46]$ & $2.57[1.95 ; 3.19]$ & $\begin{array}{l}t=-1.16 ; p=0.25 \\
W=1686 ; p=0.85\end{array}$ \\
\hline \multicolumn{4}{|l|}{ Clinical syndromes } \\
\hline Urticaria only & $37.3 \%(22 / 59)$ & $30.6 \%(22 / 72)$ & $Z=0.63, p=0.5$ \\
\hline Urticaria + Quincke's edema & $40.7 \%(24 / 59)$ & $23.6 \%(17 / 72)$ & $Z=1.9, p=0.059$ \\
\hline Quincke’s edema only & $3.4 \%(2 / 59)$ & $0.0 \%(0 / 72)$ & \\
\hline Other skin rashes only & $13.6 \%(8 / 59)$ & $20.8 \%(15 / 72)$ & $Z=0.86, p=0.39$ \\
\hline Other skin rashes + Quincke's edema & $5.1 \%(3 / 59)$ & $18.1 \%(13 / 72)$ & $Z=1.99, p=0.049$ \\
\hline Anaphylaxis & $0.0 \%(0 / 59)$ & $6.9 \%(5 / 72)$ & \\
\hline Urticaria ВсеГо & $78.0 \%(46 / 59)$ & $54.2 \%(39 / 72)$ & $Z=2.7, p=0.009$ \\
\hline Other skin rashes ВсеГо & $18.6 \%(11 / 59)$ & $38.9 \%(28 / 72)$ & $Z=2.33, p=0.022$ \\
\hline
\end{tabular}

${ }^{a}$ Statistical criteria: $t$ : Student's $t$-test; KWT: Kruskal-Wallis Test; $\chi^{2}$ : Pearson's chi-square with Yates correction.

Patients of $\mathrm{FH}$ and $\mathrm{DH}$ groups were comparable in age $(t=0.18 ; p=0.86 ; W=2038.5 ; p=0.7)$, and in the $\mathrm{FH}$ group, $62.7 \%(37 / 59)$ were boys, $p=0.0054$. The clinical characteristics of the patients are presented in Table 1.

Among clinical syndromes, cases of anaphylaxis were $6.9 \%$ $(5 / 72)$ in patients with hypersensitivity to drugs. Among children with food hypersensitivity, there are no patients with these syndromes. In all children with acute FHR (100.0\%; 59/59) and in most children with acute DHR (95.8\%; 69/72), skin manifestations of hypersensitivity were noted. These abundances are consistent with the literature data [12-14].

Diagnosis of monosymptom acute urticaria was equally frequent in $\mathrm{FH}$ and DH groups, $p=0.5 .40 .7 \%$ (24/59) of the patients with FHR had a combination of urticaria with Quincke's edema. This combination was more often in patients with DHR (23.6\%; 17/72), $p=0.059$. At the same time, acute urticaria was diagnosed in $64.9 \%(85 / 131)$ of the patients, more often in children with FHR $(78.0 \%$; 46/59) versus DHR (54.2\%; 39/72), $p=0.009$.

Various skin rashes were observed in $18.6 \%$ (11/59) of the patients of the $\mathrm{FH}$ group versus $38.9 \%(28 / 72)$ in the $\mathrm{DH}$ group, $p=0.022$. A combination of various skin rashes with Quincke's edema took place in 5.1\% (3/59) of the patients with FHR versus $18.1 \%(13 / 72)$ of the patients in the DHR group, $p=0049$.

In our study, in the vast majority of patients, hypersensitivity reactions developed after 6 hours from the exposure to the alleged causative antigen. We believe that many of the immediate reactions were stopped at the prehospital stage by emergency doctors; the need for hospitalization, apparently, occurred mainly in patients with nonimmediate reactions.

In the group of patients with drug hypersensitivity, the development of symptoms during the first 6 hours after taking the drug occurred in $8.3 \%(6 / 72)$ patients (mean time $0.25 \pm 0.29[-0.04 ; 0.54]$ hours). Among them, $6.9 \%$ $(5 / 72)$ patients had anaphylaxis. Single patient, $1.4 \%(1 / 72)$, had skin rashes that debuted as urticaria, but subsequently, a prolonged course of rashes with the formation of pigmentation was noted. Delayed (after 6 hours) onset of symptoms of HSR to drugs was noted in $91.7 \%(66 / 72)$ of the patients. Among them, in $15.3 \%(11 / 72)$ of the patients, hypersensitivity reactions formed in the range from 6 to 24 hours after the last dose of drugs, and in $76.4 \%$ (55/72) of the patients, they formed after 24 hours after the last dose of medication.

In the group of patients with HSR to food, the development of symptoms during the first 6 hours after eating was observed in $18.6 \%(11 / 59)$ of the patients (mean time $0.82 \pm 0.48[0.34 ; 1.3]$ hours). Symptoms included urticaria in $6.8 \%(4 / 59)$ of the patients, angioedema in $3.4 \%(2 / 59)$ of the patients, and their combination in $8.5 \%(5 / 59)$ of the children. Delayed (after 6 hours) onset of symptoms of hypersensitivity to food occurred in $81.4 \%(48 / 59)$ of the patients, including within 6-24 hours in $64.4 \%(38 / 59)$ of the children and after 24 hours in $17.0 \%(10 / 59)$ of the patients.

All children received complex treatment, including syndrome therapy, detoxification treatment, systemic glucocorticosteroids, and antihistamines. Doses of systemic glucocorticosteroids administered to a child before taking blood for general clinical analysis were taken into account. More often blood sampling was carried out on the first day of therapy. Doses of systemic glucocorticosteroids were $2.18 \pm 0.28[1.90 ; 2.46]$ and $2.57 \pm 0.61[1.95 ; 3.19]$ milligrams per kilogram of child weight according to prednisolone with hypersensitivity to food and drugs, respectively (without differences between groups, $p=0.85$ ). 
TABLE 2: Basic hematological values in children with acute food- and drug-induced hypersensitivity reactions.

\begin{tabular}{|c|c|c|c|}
\hline \multirow{2}{*}{ Parameters } & \multicolumn{2}{|c|}{ Group of patients } & \multirow[b]{2}{*}{ Statistics } \\
\hline & FH group & $\mathrm{DH}$ group & \\
\hline Number of patients & 59 & 72 & \\
\hline RBC, 1012/l & $4.51[4.41 ; 4.62]$ & $4.30[4.21 ; 4.38]$ & $\begin{array}{c}t=3.27, p=0.0014 \\
W=1483.0, p=0.0023\end{array}$ \\
\hline RBC, 1012/l, boys & $4.59[4.47 ; 4.72]$ & $4.31[4.17 ; 4.46]$ & $\begin{array}{c}t=3.0, p=0.004 \\
W=398.5, p=0.005\end{array}$ \\
\hline RBC, 1012/l, girls & $4.37[4.19 ; 4.55]$ & $4.28[4.18 ; 4.38]$ & $\begin{array}{c}t=0.98, p=0.33 \\
W=359.5, p=0.44\end{array}$ \\
\hline $\mathrm{HB}, \mathrm{g} / \mathrm{L}$ & $135.1[132.2 ; 138.0]$ & $128.4[126.2 ; 130.6]$ & $\begin{array}{c}t=3.7, p=0.0003 \\
W=1451.0, p=0.0009\end{array}$ \\
\hline $\mathrm{HB}, \mathrm{g} / \mathrm{L}$, boys & $136.6[132.8 ; 140.4]$ & $128.1[124.3 ; 132.0]$ & $\begin{array}{c}t=3.17, p=0.002 \\
W=402.5, p=0.006\end{array}$ \\
\hline $\mathrm{HB}, \mathrm{g} / \mathrm{L}$, girls & $132.5[127.9 ; 137.2]$ & $128.6[126.0 ; 131.3]$ & $\begin{array}{c}t=1.62, p=0.11 \\
W=323.5, p=0.11\end{array}$ \\
\hline RHB & $1.029[1.010 ; 1.048]$ & $0.988[0.97 ; 1.003]$ & $\begin{array}{c}t=3.46, p=0.0007 \\
W=1496.5, p=0.0019\end{array}$ \\
\hline RHB boys & $1.029[1.006 ; 1.052]$ & $0.985[0.960 ; 1.010]$ & $\begin{array}{c}t=2.6, p=0.012 \\
W=454.0, p=0.029\end{array}$ \\
\hline RHB girls & $1.029[0.993 ; 1.064]$ & $0.991[0.975 ; 1.007]$ & $\begin{aligned} t & =2.26, p=0.028 \\
W & =294.5, p=0.044\end{aligned}$ \\
\hline HCT, \% & $39.13[38.33 ; 39.92]$ & $37.77[37.08 ; 38.46]$ & $\begin{array}{c}t=2.58, p=0.011 \\
W=1327.5, p=0.015\end{array}$ \\
\hline $\mathrm{MCV}, \mathrm{fl}$ & 81.07 [80.08; 82.06] & 81.14 [79.99; 82.31] & $\begin{array}{c}t=-0.09, p=0.9 \\
W=1846.5, p=0.9\end{array}$ \\
\hline $\mathrm{MCH}, \mathrm{pg}$ & $27.84[27.37 ; 28.32]$ & $27.93[27.33 ; 28.54]$ & $\begin{array}{c}t=-0.23, p=0.82 \\
W=1765.5, p=0.75\end{array}$ \\
\hline PLT, 109/1 & $294.54[273.87 ; 315.20]$ & $296.35[279.09 ; 313.61]$ & $\begin{array}{c}t=-0.14, p=0.89 \\
W=2033.5, p=0.83\end{array}$ \\
\hline RDW-SD, fl & $38.7[37.76 ; 39.65]$ & $38.93[38.00 ; 39.85]$ & $\begin{array}{c}t=-0.33, p=0.75 \\
W=1860.5, p=0.76\end{array}$ \\
\hline RDV-CV, \% & $13.40[13.14 ; 13.67]$ & $13.50[13.24 ; 13.76]$ & $\begin{array}{c}t=-0.53, p=0.6 \\
W=1965.0, p=0.4\end{array}$ \\
\hline PDW, \% & $12.67[11.98 ; 13.35]$ & $11.85[11.40 ; 12.30]$ & $\begin{array}{c}t=2.08, p=0.039 \\
W=1467.5, p=0.063\end{array}$ \\
\hline MPV, fl & $10.62[10.30 ; 10.95]$ & $10.29[10.01 ; 10.56]$ & $\begin{array}{c}t=1.57, p=0.12 \\
W=1530.0, p=0.12\end{array}$ \\
\hline P-lcr, \% & $30.37[28.00 ; 32.75]$ & $27.41[25.45 ; 29.37]$ & $\begin{array}{c}t=1.94, p=0.054 \\
W=1394.5, p=0.069\end{array}$ \\
\hline PCT, \% & $0.32[0.30 ; 0.34]$ & $0.31[0.29 ; 0.33]$ & $\begin{array}{c}t=0.718835, p=0.47 \\
W=1710.5, p=0.54\end{array}$ \\
\hline WBC, $109 / 1$ & $10.42[9.47 ; 11.38]$ & $11.06[10.13 ; 11.99]$ & $\begin{array}{c}t=-0.95, p=0.35 \\
W=2307.0, p=0.6\end{array}$ \\
\hline WBC/WBCmed & $1.58[1.43 ; 1.73]$ & $1.67[1.51 ; 1.82]$ & $\begin{array}{c}t=-0.79, p=0.4 \\
W=1924.5, p=0.65\end{array}$ \\
\hline NEUT, \% & $77.23[73.77 ; 80.807]$ & 74.9677 [71.437; 78.49] & $\begin{array}{c}t=0.92, p=0.36 \\
W=1772.5, p=0.41\end{array}$ \\
\hline NEUT, abs, 109/1 & $8.28[7.29 ; 9.28]$ & $8.51[7.44 ; 9.57]$ & $\begin{array}{c}t=-0.30, p=0.77 \\
W=1450.5, p=0.75\end{array}$ \\
\hline LYM, \% & $18.53 \pm 2.99[15.52 ; 21.51]$ & $20.47[17.54 ; 23.41]$ & $\begin{array}{c}t=-0.92, p=0.36 \\
W=2120.0, p=0.29\end{array}$ \\
\hline LYM, abs, 109/1 & $1.76[1.43 ; 2.09]$ & $2.11[1.77 ; 2.45]$ & $\begin{array}{c}t=-1.45, p=0.15 \\
W=1744.0, p=0.06\end{array}$ \\
\hline MONO, \% & $3.74[3.08 ; 4.40]$ & $4.16[3.34 ; 4.98]$ & $\begin{array}{c}t=-0.77, p=0.44 \\
W=1980.0, p=0.84\end{array}$ \\
\hline MONO, abs, 109/1 & $0.369[0.29 ; 0.45]$ & $0.47[0.37 ; 0.58]$ & $\begin{array}{c}t=-1.57677, p=0.12 \\
W=1687.5, p=0.37\end{array}$ \\
\hline $\mathrm{EO}, \%$ & $0.37[0.15 ; 0.60]$ & $0.50[0.13 ; 0.88]$ & $\begin{array}{c}t=-0.57, p=0.57 \\
W=1661.5, p=0.97\end{array}$ \\
\hline EO, abs, 109/1 & $0.029[0.008 ; 0.050]$ & $0.060[0.021 ; 0.010]$ & $\begin{array}{c}t=-1.26, p=0.21 \\
W=1691.0, p=0.22\end{array}$ \\
\hline
\end{tabular}


TABLE 2: Continued.

\begin{tabular}{lccc}
\hline Parameters & \multicolumn{2}{c}{ Group of patients } & Statistics \\
\hline ESR, mm/hour & $8.91[7.83 ; 10.00]$ & DH group & $t=-1.61, p=0.11$ \\
& & $10.44[8.99 ; 11.88]$ & $W=2359.5, p=0.26$ \\
\hline
\end{tabular}

WBC: white blood cell count; RBC: red blood cell count; HGB: level of hemoglobin molecules; HCT: hematocrit; MCV: mean corpuscular volume of red blood cells; MCH: mean corpuscular hemoglobin; PLT: platelets levels in the blood; NEUT, \%: neutrophil granulocyte, \%; MONO, \%: monocyte, \%; LYM, \%: lymphocyte, \%; EO, \%: eosinophil granulocyte, \%; BASO, \%: basophil granulocyte, \%; RDW-SD: red cell distribution width, standard deviation; RDW-CV: red cell distribution width, coefficient of variation; PDW: width of distribution of thrombocytes; MPV: mean platelet volume; P-LCR: platelet large cell ratio; PCT: plateletcrit; volume \% of platelets; ESR: erythrocyte sedimentation rate; abs.: absolute cells count, WBC/WBCmed: ratio of WBC patient to median WBC values characteristic for a given age and sex.

2.4. Laboratory Tests. All children were analyzed within 24 hours after the admission using the evaluation of hemogram, evaluation of the serum level of immunoglobulins, including the content of total immunoglobulin E, and evaluation of the main biochemical markers. The hemogram evaluation was performed on an automatic hematological analyzer of the XS series (XS-1000i/XS-800i, SYSMEX CORPORATION, Japan). Determination of the level of serum immunoglobulin $\mathrm{E}$ was performed using the IgEELISA-Best test systems, production of Vector-Best JSC, Russia, on the automated enzyme immunoassay analyzer ALISEI-QS, RADIM GROUP, Italy. The main biochemical parameters were determined on the automatic biochemical analyzer Konelab 20XT Thermo Fisher, Finland, using biochemical sets from the same manufacturer. The reference values of hematological and biochemical parameters were corrected by the sex and age of the patients. In 67 patients ( 31 in the FH group and 36 in the $\mathrm{DH}$ group), hemogram evaluation was performed twice: on the day of admission and 7-10 days later.

2.5. Statistical Analyses. Statistical differences between two and more groups were analyzed on the basis of several statistical tests. Student's $t$-test was applied for testing the null hypothesis that two population means are equal. Pearson's $\chi^{2}$ test was used for testing a relationship between two categorical variables. The Wilcoxon criterion (W) was used to test the equality of the medians of two independent samples. In all tests, $p$ values less than 0.05 were considered statistically significant. Data in tables are presented as mean value \pm standard deviation supplemented, in some cases, by 95\% confidence interval $\left[x_{\min }, x_{\max }\right]$. Statgraphics v.50 statistical software (Statpoint Technologies, Inc., USA) was used for statistical analysis.

Variables with normal distribution were expressed as the mean \pm standard deviation or percentage (\%). Variables with no normal distribution were expressed by the median value. Continuous variables were analyzed by $t$-test or Mann-Whitney test. Categorical data were analyzed using the chi-square or Fisher's exact test. To identify the risk factors related to severe anaphylaxis, multiple logistic regression analysis was used. Variables that are clinically important and those $p$ value $<0.1$ in the univariate analysis were adjusted in the multiple variate analysis. Statistical analyses were performed using Statgraphics version 18 (2017 Statgraphics Technologies, Inc.). Statistical significance was considered for $p$ values $<0.05$.

\section{Results}

3.1. Basic Hematological Values. The results of the hematological evaluation of patients are presented in Table 2. As a whole, the mean values of the main hematological parameters did not go beyond the reference limits typical for the sex and age of specific patients. However, within the considered groups, children with acute drug-induced allergic reactions showed a lower content of hemoglobin and erythrocytes in peripheral blood compared to children who had acute food-induced allergic reactions; the differences were statistically significant. As was expected, it is accompanied by lower values of hematocrit in children with druginduced acute allergic reactions, $p=0.011$. Differences are also seen in the analysis of relative values, e.g., in the ratio of the child's hemoglobin values to the median hemoglobin values characteristic for a given age and sex (RHB), $p=0.0007$.

As can be seen from Table 2, differences in the values characterizing the morphological features of erythrocytes (MCV, RDW-SD, and RDV-SV) and the hemoglobin content in erythrocytes $(\mathrm{MCH})$ between these two groups of children are not found, and the indices are within reference limits. This may indicate that iron deficiency cannot be a cause for a lower hemoglobin and erythrocyte count in patients with acute DHR compared with patients with acute HR of food genesis. The deficiency of folic acid or cyanocobalamin is also unlikely since the size of erythrocytes is not shifted towards large values. On the contrary, the MCV level is close to the lower limit of the norm, which indicates a tendency to microcytosis of erythrocytes in both acute hypersensitivity reactions in children.

The content of platelets in patients with drug and food genesis of acute HR was not significantly distinguished, $p=0.89$. In the literature data, patients with moderate and severe forms of chronic spontaneous urticaria have increased platelet count and MPV although these parameters did not exceed the limits of reference values [15]. Thrombocytopenia (lower than 150 thousand $/ \mathrm{mL}$ ) was not detected in any patients of both groups. The main parameters characterizing the morphological features of platelets were not statistically different in the children of both groups. However, there was a tendency to lower mean platelet volume (MPV) and the large cell ratio platelets (p-lcr) in children with a drug-induced hypersensitivity. The calculated width of the distribution of thrombocytes in children with drug hypersensitivity reactions was also lower than that 
TABLE 3: Dynamics hemogram in children with acute food and drug-induced hypersensitivity reactions.

\begin{tabular}{|c|c|c|c|c|}
\hline \multirow{2}{*}{ Parameters } & \multicolumn{3}{|c|}{ Group of patients } & \multirow[b]{2}{*}{ Statistics } \\
\hline & & First day & After & \\
\hline $\operatorname{RBC}\left(10^{12} / 1\right)$ & $\begin{array}{l}n=65 \\
\text { FH group, } n=31 \\
\text { DH group, } n=34\end{array}$ & $\begin{array}{l}4.40[4.29 ; 4.50] \\
4.49[4.33 ; 4.65] \\
4.31[4.18 ; 4.45]\end{array}$ & $\begin{array}{l}4.52[4.42 ; 4.63] \\
4.63[4.45 ; 4.81] \\
4.42[4.30 ; 4.54]\end{array}$ & $\begin{array}{c}t=-2.96, p=0.0043 \\
t=-2.03, p=0.05 \\
t=-2.15, p=0.039\end{array}$ \\
\hline $\mathrm{HB}(\mathrm{g} / \mathrm{L})$ & $\begin{array}{l}n=65 \\
\text { FH group, } n=31 \\
\text { DH group, } n=34\end{array}$ & $\begin{array}{l}131.11[128.12 ; 134.10] \\
134.71[129.89 ; 139.53] \\
127.82[124.30 ; 131.34]\end{array}$ & $\begin{array}{l}136.19[133.44 ; 138.93] \\
138.35[133.96 ; 142.74] \\
134.21[130.72 ; 137.69]\end{array}$ & $\begin{array}{c}t=-4.96, p=0.000005 \\
t=-2.35, p=0.03 \\
t=-4.78,=0.00003\end{array}$ \\
\hline MCV (fl) & $\begin{array}{l}n=65 \\
\text { FH group, } n=31 \\
\text { DH group, } n=34\end{array}$ & $\begin{array}{c}81.89[80.69 ; 83.09] \\
81.7[80.23 ; 83.17] \\
82.05[80.14 ; 83.95]\end{array}$ & $\begin{array}{l}81.68[80.54 ; 82.82] \\
81.14[79.81 ; 82.47] \\
82.17[80.30 ; 84.03]\end{array}$ & $\begin{array}{l}t=0.84, p=0.40 \\
t=1.34, p=0.19 \\
t=0.08, p=0.94\end{array}$ \\
\hline $\mathrm{MCH}(p g)$ & $\begin{array}{c}n=65 \\
\text { FH group, } n=31 \\
\text { DH group, } n=34\end{array}$ & $\begin{array}{l}27.88[27.30 ; 28.46] \\
27.76[27.02 ; 28.51] \\
27.98[27.08 ; 28.88]\end{array}$ & $\begin{array}{l}28.20[27.52 ; 28.88] \\
28.13[27.25 ; 29.00] \\
28.27[27.19 ; 29.34]\end{array}$ & $\begin{array}{l}t=-1.42, p=0.16 \\
t=-1.15, p=0.26 \\
t=-0.89, p=0.38\end{array}$ \\
\hline $\operatorname{PLT}\left(10^{9} / 1\right)$ & $\begin{array}{l}n=65 \\
\text { FH group, } n=31 \\
\text { DH group, } n=36\end{array}$ & $\begin{array}{l}297.18[277.69 ; 316.66] \\
291.83[259.69 ; 323.96] \\
301.88[276.99 ; 326.76]\end{array}$ & $\begin{array}{l}298.52[279.48 ; 317.55] \\
285.79[257.13 ; 314.46] \\
309.69[283.48 ; 335.91]\end{array}$ & $\begin{array}{c}t=-0.16, p=0.87 \\
t=0.21, p=0.84 \\
t=-0.41, p=0.68\end{array}$ \\
\hline $\operatorname{MPV}(f l)$ & $\begin{array}{l}n=65 \\
\text { FH group, } n=31 \\
\text { DH group, } n=36\end{array}$ & $\begin{array}{c}10.52[10.22 ; 10.81] \\
10.94[10.54 ; 11.34] \\
10.25[9.90 ; 10.59]\end{array}$ & $\begin{array}{c}10.27[10.01 ; 10.54] \\
10.75[10.32 ; 11.18] \\
9.86[9.59 ; 10.12]\end{array}$ & $\begin{array}{c}t=3.35, p=0.0015 \\
t=2.07, p=0.049 \\
t=2.72, p=0.01\end{array}$ \\
\hline P-lcr (\%) & $\begin{array}{l}n=65 \\
\text { FH group, } n=31 \\
\text { DH group, } n=34\end{array}$ & $\begin{array}{l}28.85[26.64 ; 31.07] \\
32.07[29.02,35.12] \\
26.21[23.50 ; 28.91]\end{array}$ & $\begin{array}{l}27.13[25.13 ; 29.12] \\
30.88[27.87 ; 33.89] \\
23.86[21.68,26.04]\end{array}$ & $\begin{array}{c}t=2.41, p=0.019 \\
t=2.04, p=0.05 \\
t=1.49, p=0.15\end{array}$ \\
\hline WBC (abs, 109/l) & $\begin{array}{l}n=65 \\
\text { FH group, } n=31 \\
\text { DH group, } n=34\end{array}$ & $\begin{array}{c}11.47[10.42 ; 12.51] \\
11.27[9.67 ; 12.88] \\
11.65[10.20 ; 13.09]\end{array}$ & $\begin{array}{l}10.61[9.69 ; 11.52] \\
10.74[9.18 ; 12.29] \\
10.49[9.36 ; 11.61]\end{array}$ & $\begin{array}{l}t=1.51, p=0.15 \\
t=0.61, p=0.54 \\
t=1.55, p=0.13\end{array}$ \\
\hline NEUT (abs, $\left.10^{9} / \mathrm{l}\right)$ & $\begin{array}{l}n=65 \\
\text { FH group, } n=31 \\
\text { DH group, } n=34\end{array}$ & $\begin{array}{l}9.03[7.94 ; 10.11] \\
9.42[7.90 ; 10.94] \\
8.68[7.08 ; 10.29] \\
\end{array}$ & $\begin{array}{l}5.14[4.42 ; 5.86] \\
5.15[4.02 ; 6.28] \\
5.13[4.15 ; 6.11] \\
\end{array}$ & $\begin{array}{c}t=6.09 p=1.37352 E-7 \\
t=3.77, p=0.001 \\
t=4.75, p=0.00005\end{array}$ \\
\hline LYM (abs, $\left.10^{9} / 1\right)$ & $\begin{array}{l}n=65 \\
\text { FH group, } n=31 \\
\text { DH group, } n=34\end{array}$ & $\begin{array}{l}2.05[1.70 ; 2.40] \\
1.78[1.35 ; 2.20] \\
2.28[1.74 ; 2.81] \\
\end{array}$ & $\begin{array}{l}4.05[3.58 ; 4.51] \\
3.83[3.13 ; 4.53] \\
4.23[3.59 ; 4.87] \\
\end{array}$ & $\begin{array}{c}t=-7.07, p=4.46932 E-7 \\
t=-5.65, p=0.000008 \\
t=-4.53, p=0.00008\end{array}$ \\
\hline MONO (abs, $\left.10^{9} / \mathrm{l}\right)$ & $\begin{array}{l}n=65 \\
\text { FH group, } n=31 \\
\text { DH group, } n=34\end{array}$ & $\begin{array}{l}0.46[0.36 ; 0.56] \\
0.42[0.29 ; 0.56] \\
0.48[0.34 ; 0.63] \\
\end{array}$ & $\begin{array}{l}0.81[0.73 ; 0.89] \\
0.88[0.74 ; 1.02] \\
0.76[0.66 ; 0.85] \\
\end{array}$ & $\begin{array}{c}t=-6.32, p=5.23158 E-8 \\
t=-6.48, p=0.000001 \\
t=-3.29, p=0.0025\end{array}$ \\
\hline EO (abs, $\left.10^{9} / \mathrm{l}\right)$ & $\begin{array}{l}n=65 \\
\text { FH group, } n=31 \\
\text { DH group, } n=34\end{array}$ & $\begin{array}{l}0.044[0.013 ; 0.075] \\
0.023[0.007 ; 0.038] \\
0.061[0.005 ; 0.117]\end{array}$ & $\begin{array}{l}0.270[0.209 ; 0.331] \\
0.298[0.207 ; 0.389] \\
0.247[0.159 ; 0.334]\end{array}$ & $\begin{array}{c}t=-6.65, p=1.53913 E-8 \\
t=-5.43, p=0.000016 \\
t=-4.15, p=0.0002\end{array}$ \\
\hline
\end{tabular}

WBC: white blood cell count; RBC-red blood cell count; HGB: level of hemoglobin molecules; HCT: hematocrit; MCV: mean corpuscular volume of red blood cells; MCH: mean corpuscular hemoglobin; PLT: platelet levels in the blood; NEUT: neutrophil granulocyte; MONO: monocyte; LYM: lymphocyte; EO: eosinophil granulocyte; MPV: mean platelet volume; P-LCR: platelet large cell ratio; abs.: absolute cells count.

in patients with food reactions. Thus, in patients with hypersensitive reactions to drugs, the size of platelets (more mature forms) has a tendency to decrease, which suggests active platelet degranulation in this patient population.

Differences in the leukocyte count were not observed, and neutrophilia was typical for patients of both groups, which was apparently due to the use of systemic glucocorticosteroids on the first day of therapy $(2.18 \pm 0.28$ in $\mathrm{FH}$ group and $2.57 \pm 0.61 \mathrm{mg} / \mathrm{kg}$ in DH group on average according to prednisolone). Patients with drug genesis of acute hypersensitivity reactions have a high level of lymphocytes and monocytes, but this difference was not significantly distinguished ( $p=0.36$ and $p=0.44)$. But this tendency supports an opinion about recent infectious diseases at this group of patients.
Hemogram evaluation performed during 7-10 days after the admission demonstrated a higher level of hemoglobin in both groups. In parallel, MCV and $\mathrm{MCH}$ were equal.

Platelet count was equal, with decreased platelet size, especially in the DH group $(10.25 \pm 0.35$ versus $9.86 \pm 0.26$, $t=2.72, p=0.01)$. It may be concerned with increased thrombopoietin synthesis in patients with drug-induced hypersensitivity reactions (associated with antibiotic use due to infectious process); see Table 3.

White blood count was equal in both groups, with reduced neutrophils and relevant increased levels of lymphocytes, monocytes, and eosinophils. These changes may be induced by the use of glucocorticosteroids in high doses. It should be noted that, in the FH group, the increase of eosinophils after 7-10 days was more significant (from $0.023 \pm 0.02$ to $0.298 \pm 0.091$, $p=0.00002)$, remaining within the normal range, Table 3 . 
TABLE 4: Biochemical parameters of peripheral venous blood in patients with food- and drug-induced hypersensitivity reactions.

\begin{tabular}{|c|c|c|c|}
\hline Biochemical parameter & FH group & $\mathrm{DH}$ group & Statistics \\
\hline $\operatorname{ALAT}(\mu \mathrm{cat} / \mathrm{L})$ & $0.32[0.29 ; 0.36]$ & $0.39[0.32 ; 0.45]$ & $\begin{aligned} t & =-1.6 ; p=0.12 \\
W & =2191.0 ; p=0.51\end{aligned}$ \\
\hline $\operatorname{ASAT}(\mu \mathrm{cat} / \mathrm{L})$ & $0.74[0.64 ; 0.83]$ & $0.62[0.55 ; 0.70]$ & $\begin{array}{c}t=2.07 ; p=0.04 \\
W=1710.0 ; p=0.09\end{array}$ \\
\hline ASAT/ALAT de Ritis ratio & $2.47[0.430 ; 0.67]$ & $0.83[0.597 ; 1.07]$ & $\begin{array}{c}t=-1.98 ; p=0.049 \\
W=2743.0 ; p=0.004\end{array}$ \\
\hline Glucose (mmol/l) & $5.46[5.16 ; 5.76]$ & $5.44[5.18 ; 5.71]$ & $\begin{array}{c}t=0.07 ; p=0.94 \\
W=1969.0 ; p=0.22\end{array}$ \\
\hline Creatinine $(\mu \mathrm{mol} / \mathrm{L})$ & $63.54[59.64 ; 67.45]$ & $61.89[58.60 ; 65.19]$ & $\begin{array}{c}t=0.65 ; p=0.52 \\
W=1996.5 ; p=0.79\end{array}$ \\
\hline Urea $(\mathrm{mmol} / \mathrm{l})$ & $5.28[4.87 ; 5.69]$ & $4.79[4.45 ; 5.14]$ & $\begin{array}{c}t=1.82 ; p=0.071 \\
W=1634 ; p=0.055\end{array}$ \\
\hline GFR $(\mathrm{ml} / \mathrm{min})$ & $111.1[103.35 ; 118.85]$ & $110.1[105.84 ; 116.13]$ & $\begin{array}{c}t=0.02 ; p=0.98 \\
W=1202.0 ; p=0.69\end{array}$ \\
\hline Urea/creatinine ratio & $85.30[77.56 ; 93.04]$ & $82.34[75.66 ; 89.03]$ & $\begin{array}{c}t=0.58 ; p=0.56 \\
W=1921.5 ; p=0.59\end{array}$ \\
\hline $\mathrm{AP}(\mathrm{E} / \mathrm{l})$ & $513.40[453.64 ; 573.28]$ & $392.60[344.78 ; 440.49]$ & $\begin{array}{c}t=3.2 ; p=0.002 \\
W=321.5 ; p=0.007\end{array}$ \\
\hline Bilirubin $(\mu \mathrm{mol} / \mathrm{L})$ & $11.34[9.71 ; 12.98]$ & $10.48[9.11 ; 11.86]$ & $\begin{array}{c}t=0.81 ; p=0.42 \\
W=1605.5 ; p=0.24\end{array}$ \\
\hline $\operatorname{IgE}(\mathrm{E} / \mathrm{l})$ & $131.16[88.59 ; 173.74]$ & $124.76[82.95 ; 166.57]$ & $\begin{array}{c}t=0.21 ; p=0.83 \\
W=1933.0 ; p=0.87\end{array}$ \\
\hline
\end{tabular}

ASAT: aspartate aminotransferase; ALAT: alanine aminotransferase; AP: alkaline phosphatase; CFR: glomerular filtration rate; IgE: immunoglobulin E.

3.2. Biochemical Markers, Reflecting the Functioning of the Hepatobiliary System. The main biochemical parameters measured in $\mathrm{FH}$ and $\mathrm{DH}$ groups of patients are shown in Table 4. As is seen from the table, no significant differences between groups were found. However, some interesting features should be noted in these results.

Levels of aspartate aminotransferase (ASAT), alanine aminotransferase (ALAT), and lactate dehydrogenase were comparable in both groups, and mean values and confidence intervals of these parameters did not exceed the limits of reference values. However, in the analysis of individual indicators, it was noted that insignificant excess of ASAT level was somewhat more frequent $(39.7 \% ; 25 / 63)$ in patients with hypersensitive reactions to food, and the corresponding levels were in the range from 0.15 to $1.64 \mu \mathrm{cat} / \mathrm{L}$. At the same time, among patients with hypersensitive reactions to drugs, the increased levels of ASAT occurred in $24.6 \%$ (17/69) of cases, ranging from 0.09 to $1.45 \mu \mathrm{cat} / \mathrm{L}$. One patient with food hypersensitivity $(0.98 \mu \mathrm{cat} / \mathrm{L})$ and $10.1 \%(7 / 69)$ of the patients with drug hypersensitivity had increased ALAT level $(0.11-1.93 \mu \mathrm{cat} / \mathrm{L})$. Thus, cytolysis syndrome is not abundant in children with clinically manifested acute drug hypersensitivity, and it occurred in less than a quarter of patients. It should be noted that, as has been suggested recently, aminotransferases widely used in the classification of cytolysis markers are perhaps insufficiently informative for the diagnosis of acute liver damage. [16].

The level of alkaline phosphatase (AP) and total bilirubin in the blood serum was significantly higher in patients with acute allergic reactions provoked by food. An increase in alkaline phosphatase (above $500 \mathrm{U} / \mathrm{L}$, ranging from 501 to $822 \mathrm{U} / \mathrm{L})$ was observed in $28.6 \%(12 / 42)$ of the children in the
DH group. In the FH group, the increase in AP was observed in $48.3 \%(14 / 29)$ of the children. Perhaps, this indicates a characteristic cholestasis for this group of patients. The level of bilirubin was exceeded in $14.04 \%$ (8/57), ranging from 20.4 to $31.1 \mu \mathrm{mol} / \mathrm{L}$, in the $\mathrm{FH}$ group and in two patients $(3.08 \% ; 2 / 65)$ in $\mathrm{DH}$ group. Given that the level of bilirubin is an indirect marker of hemolysis, the absence of its increase in children with hypersensitivity to drugs does not support the assumption that the hemolysis is the leading cause of a lower hemoglobin level in these children.

3.3. Biochemical Parameters That Reflect the Filtration Function of the Kidneys. Levels of serum creatinine, urea of peripheral blood, and GFR were comparable in both groups of patients and did not exceed the reference limits. It should be noted, however, that these indicators are not currently considered as early markers of acute kidney damage, and this issue requires the measurements of minute diuresis, cystatin $\mathrm{C}$, and enzyme activity in urine.

The analysis of the humoral immunity parameters indicates that significant differences in the level of serum content of total $\operatorname{IgE}$ in patients of both groups are not observed, which indicates a comparable contribution of mechanisms associated with atopy to the genesis of acute hypersensitivity reactions provoked by both food and drugs.

It is interesting that patients with urticaria have a higher level of hemoglobin and RBC. We can suppose that in case other skin rashes with hyperpigmentation take place deposition of iron in macrophages can be found with depressed amounts of iron for erythropoiesis, like anemia of chronic disease. Deposition of iron in histiocytes of the lower dermis 
TABLE 5: Basic hematological values in children with different skin manifestations of acute food- and drug-induced hypersensitivity reactions.

\begin{tabular}{|c|c|c|c|}
\hline \multirow{2}{*}{ Parameters } & \multicolumn{2}{|c|}{ Group of patients } & \multirow[b]{2}{*}{ Statistics } \\
\hline & UT group & Other rashes group & \\
\hline Number of patients & 86 & 40 & 126 \\
\hline $\mathrm{RBC}\left(10^{12} / \mathrm{l}\right)$ & $4.47[4.38 ; 4.55]$ & $4.24[4.13 ; 4.35]$ & $\begin{array}{c}t=3.09 ; p=0.002 \\
W=1167.5 ; p=0.005\end{array}$ \\
\hline RBC boys & $\begin{array}{c}4.56[4.43 ; 4.69] \\
n=45\end{array}$ & $\begin{array}{c}4.29[4.17 ; 4.42] \\
n=23\end{array}$ & $\begin{array}{c}t=2.68 ; p=0.009 \\
W=318.5 ; p=0.01\end{array}$ \\
\hline RBC girls & $\begin{array}{c}4.36[4.20 ; 4.46] \\
n=41\end{array}$ & $\begin{array}{c}4.17[3.98 ; 4.37] \\
n=17\end{array}$ & $\begin{array}{c}t=1.86 ; p=0.068 \\
W=243.0 ; p=0.09\end{array}$ \\
\hline $\mathrm{HB}(\mathrm{g} / \mathrm{L})$ & $133.62[131.39 ; 135.84]$ & $127.0[123.82 ; 130.18]$ & $\begin{array}{c}t=3.38 ; p=0.001 \\
W=1138.0 ; p=0.002\end{array}$ \\
\hline HB boys & $135.11[131.66 ; 138.56]$ & $128.17[123.50 ; 132.84]$ & $\begin{aligned} t & =2.40 ; p=0.019 \\
W & =353.0 ; p=0.033\end{aligned}$ \\
\hline HB girls & $131.98[129.18 ; 134.77]$ & $125.41[120.94 ; 129.88]$ & $\begin{array}{c}t=2.58 ; p=0.012 \\
W=218.0 ; p=0.026\end{array}$ \\
\hline RHB & $1.021[1.007 ; 1.036]$ & $0.977[0.957 ; 0.996]$ & $\begin{aligned} t & =3.56 ; p=0.0005 \\
W & =1099.5 ; p=0.001\end{aligned}$ \\
\hline RHB boys & $1.025[1.004 ; 1.046]$ & $0.978[0.950 ; 1.006]$ & $\begin{aligned} t & =2.68 ; p=0.009 \\
W & =332.5 ; p=0.017\end{aligned}$ \\
\hline RHB girls & $1.017[0.991 ; 1.042]$ & $0.974[0.942 ; 1.011]$ & $\begin{aligned} t & =2.34 ; p=0.02 \\
W & =214.5 ; p=0.02\end{aligned}$ \\
\hline $\operatorname{PLT}\left(10^{9} / 1\right)$ & $296.93[280.31 ; 313.55]$ & $293.79[268.22 ; 319.36]$ & $\begin{array}{c}t=0.21 ; p=0.83 \\
W=1497.5 ; p=0.73\end{array}$ \\
\hline PDW (\%) & $12.34[11.85 ; 12.83]$ & $11.92[11.20 ; 12.65]$ & $\begin{array}{c}t=0.96 ; p=0.34 \\
W=1282.0 ; p=0.20\end{array}$ \\
\hline MPV (fl) & $10.48[10.22 ; 10.74]$ & $10.29[9.87 ; 10.71]$ & $\begin{aligned} t & =0.81 ; p=0.42 \\
W & =1315.5 ; p=0.28\end{aligned}$ \\
\hline P-lcr (\%) & $29.03[27.15 ; 30.92]$ & $27.74[24.86 ; 30.63]$ & $\begin{array}{c}t=0.77 ; p=0.44 \\
W=1254.0 ; p=0.30\end{array}$ \\
\hline WBC $\left(10^{9} / 1\right)$ & $10.69[9.89 ; 11.50]$ & $10.84[9.49 ; 12.20]$ & $\begin{array}{c}t=-0.19 ; p=0.85 \\
W=1747.5 ; p=0.89\end{array}$ \\
\hline EO (\%) & $0.33[0.13 ; 0.52]$ & $0.65[0.24 ; 1.07]$ & $\begin{array}{c}t=1.38 ; p=0.17 \\
W=1691 ; p=0.35\end{array}$ \\
\hline EO (abs, $\left.10^{9} / \mathrm{l}\right)$ & $0.028[0.008 ; 0.050]$ & $0.060[0.021 ; 0.100]$ & $\begin{array}{l}t=-1.26 ; p=0.21 \\
W=1691 ; p=0.22\end{array}$ \\
\hline ESR (mm/hour) & $10.14[8.89 ; 11.40]$ & $9.30[7.74 ; 10.86]$ & $\begin{array}{c}t=0.79 ; p=0.43 \\
W=1555.0 ; p=0.50\end{array}$ \\
\hline
\end{tabular}

WBC: white blood cell count; RBC-red blood cell count; HGB: level of hemoglobin molecules; MCV: mean corpuscular volume of red blood cells; MCH: mean corpuscular hemoglobin; PLT: platelet levels in the blood; EO, \%: eosinophil granulocyte, \%; PDW: width of distribution of thrombocytes; MPV: mean platelet volume; P-LCR: platelet large cell ratio; PCT: plateletcrit; volume \% of platelets; ESR: erythrocyte sedimentation rate, abs.

and subcutaneous fat is described by S.P. Keijmel et al. and other authors [17]. Data are presented in Table 5.

Biochemical parameters of peripheral venous blood in patients with different skin manifestations revealed an increase in alkaline phosphatase, $\mathrm{LDH}$, and De Ritis ratio. This can explain the higher percentage of patients with urticaria in the FH group. Data are presented in Table 6.

\section{Discussion}

Hypersensitivity is related to type B ADR reactions and is mainly mediated by the immune system [15]. These reactions can affect virtually any organ, but the skin, liver, and blood cells are the most common targets [18]. With respect to skin reactions, due to the availability of visual diagnostics, significant progress has been made in systematizing possible skin syndromes in acute drug-induced hypersensitivity, which is reflected in the numerous relevant publications
$[13,14,19,20]$. In contrast, the hematological features of ADR are studied to a significantly less extent. It was found that hematological changes in hypersensitivity reactions of drug origin can be characterized by cytopenic syndromes, including anemia, thrombocytopenia, and leukopenia [18]. At the same time, drug-induced immune hemolytic anemia (DIIHA) is a rare cytopenia. These cases are $10 \%$ of autoimmune anemia, whose incidence is 1 per 100 000/year and approximately half of the cases are secondary to an associated disorder [16]. This pathological condition remains insufficiently studied at the moment and the main drugs provoking hemolysis are antibacterial drugs $[16,21]$. The destruction of erythrocytes can be caused either by the direct action of the drug on the erythrocyte or indirectly through immune mechanisms [22].

Except for drug-induced hemolytic anemia, aplastic anemia can occur as a result of bone marrow injuries [23]. Its development as a symptom of hypersensitive reactions to 
TABLE 6: Biochemical parameters and total serum IgE of peripheral venous blood in patients with different skin manifestations of hypersensitivity reactions.

\begin{tabular}{|c|c|c|c|}
\hline Biochemical parameter & UT group & Other rashes group & Statistics \\
\hline $\operatorname{ALAT}(\mu \mathrm{cat} / \mathrm{L})$ & $0.32[0.29 ; 0.36]$ & $0.39[0.32 ; 0.46]$ & $\begin{array}{c}t=-1.57 ; p=0.12 \\
W=2191.0 ; p=0.55\end{array}$ \\
\hline $\operatorname{ASAT}(\mu \mathrm{cat} / \mathrm{L})$ & $0.74[0.64 ; 0.83]$ & $0.62[0.54 ; 0.69]$ & $\begin{array}{c}t=2.07 ; p=0.04 \\
W=1710.5 ; p=0.094\end{array}$ \\
\hline ASAT/ALAT De Ritis ratio & $2.47[2.7 ; 2.76]$ & $1.93[1.69 ; 2.17]$ & $\begin{aligned} t & =2.85 ; p=0.005 \\
W & =1452.0 ; p=0.004\end{aligned}$ \\
\hline $\mathrm{LDH}(\mathrm{E} / \mathrm{l})$ & $432.8[388.1 ; 477.5]$ & $410.7[357.5 ; 463.8]$ & $\begin{array}{c}t=0.57 ; p=0.57 \\
W=325.0 ; p=0.56\end{array}$ \\
\hline $\mathrm{AP}(\mathrm{E} / \mathrm{l})$ & $513.5[453.6 ; 573.3]$ & $392.4[342.0 ; 442.8]$ & $\begin{array}{c}t=3.1 ; p=0.0026 \\
W=307.5 ; p=0.0077\end{array}$ \\
\hline Bilirubin $(\mu \mathrm{mol} / \mathrm{L})$ & $11.34[9.71 ; 12.98]$ & $10.48[9.11 ; 11.86]$ & $\begin{array}{c}t=0.81 ; p=0.42 \\
W=1605.5 ; p=0.24\end{array}$ \\
\hline $\operatorname{IgE}(\mathrm{E} / \mathrm{l})$ & $131.2[88.6 ; 173.7]$ & $127.5[84.6 ; 170.4]$ & $\begin{array}{c}t=0.12 ; p=0.9 \\
W=1891.0 ; p=0.9\end{array}$ \\
\hline
\end{tabular}

ASAT: aspartate aminotransferase; ALAT: alanine aminotransferase; AP: alkaline phosphatase; CFR: glomerular filtration rate; IgE: immunoglobulin E.

drugs, including antibacterial drugs, has also been described in the literature, including its combination with skin symptoms $[5,24]$.

Among these reactions, the most attention is usually paid to the developed cytopenic and pancytopenic syndromes.

Relatively lower values of hemoglobin and erythrocyte levels in patients with acute hypersensitivity reactions to medicines may reflect important effects:

(a) Recent infectious diseases (more than half of the children had antibiotics and nonsteroidal anti-inflammatory drugs as causal drugs). In this regard, the mechanisms can be similar to the mechanisms of anemia of chronic disease and be determined by the effect of hepcidin on the components of red blood. To clarify the validity of this assumption, it is important to analyze the hemograms of these patients in the catamnesis. Decreased erythropoiesis can be a result of direct influences of proinflammatory cytokines, such as IL 6, FNO alfa, and others. These cytokines not only stimulated the production of hepcidin but also directly affected erythroid cell development $[17,25]$.

(b) Perhaps, the patients with hypersensitivity to drugs had initially lower hemoglobin values, which were reduced as a protective reaction of the organism to undesirable drug effects. There is evidence in the literature that hemoglobin has similar protective properties for some active molecules and radicals [26]. There are also observations that a decrease in hemoglobin is associated with an increased risk of developing severe hypersensitivity reactions to drugs including Stevens-Johnson syndrome in some categories of patients [27].

(c) Hidden secondary hypersensitivity to medicines influencing the hemoglobin levels, e.g., the effect of drugs on the synthesis of erythropoietin by the kidneys. This requires a detailed study of markers of acute renal damage, including the dynamics of the content of cystatin $\mathrm{C}$ and other markers. (d) Hemolysis of erythrocytes under the influence of a number of drugs. In this case, the bilirubin levels, which are an indirect marker of hemolysis, should be increased. Below, we will demonstrate that the measured values of bilirubin do not support this assumption and there is no correlation between bilirubin and hemoglobin levels.

It is known that, in addition to hemostasis, platelets play an important role in intercellular communication, mediating inflammatory, and immunomodulating processes. In this regard, the study of markers for platelet activation can be very useful for understanding the pathogenesis of drug hypersensitivity and the subsequent monitoring of these pathological processes [28]. At the same time, patients with chronic urticaria have a higher value of MPV [29], but this opinion is not suggested by all authors [30].

4.1. Limitation of Our Research. We did not evaluate the viral status of patients, but viral exanthem can clinically mimic drug exanthem [31].

\section{Conclusion}

Patients with acute hypersensitivity reactions induced by food and medicines had comparable clinical symptoms with a predominance of skin lesions. The significant differences in hematological parameters between the group of children and adolescent patients with drug- and food-induced hypersensitivity reactions were found in their red blood characteristics. In patients with hypersensitive reactions to drugs, significantly lower levels of erythrocytes and hemoglobin were found, while the median values of these parameters did not exceed the limits of reference values. These differences persisted also in the analysis of hemoglobin values, analyzed with accounting for the age and sex of the patients. The reduction of hemoglobin was not accompanied by an increase in bilirubin in these patients. Thus, this fact does not support the assumption about the drug-induced hemolysis 
as a main effect influencing the hematological parameters in these patients.

Taking into account the fact that the development of drug hypersensitivity, especially in its immune-dependent variants, is delayed (mostly from several days to several weeks), the patients whose relative hemoglobin level corrected for the age and sex on the background or after the completion of therapy is less than 1.0 should be kept observed as a special anxiety group. This is especially important for patients after antibacterial therapy.

Thus, the results indicate the various mechanisms of drug-induced hypersensitivity affecting the frequency and severity of the hypersensitivity reactions, their prognosis, and understanding of other diseases associated with environmental and/or technogenic influences.

\section{Data Availability}

The data used to support the findings of this study are available from the corresponding author upon request.

\section{Conflicts of Interest}

The authors declare that there are no potential conflicts of interest associated with this manuscript.

\section{Authors' Contributions}

All authors contributed equally to this work.

\section{Acknowledgments}

The work was performed according to the Russian Government Program of Competitive Growth of Kazan Federal University. The authors are thankful for the opportunity to present abstracts describing the preliminary results on the subject of the present study in the Allergy-European Journal of Allergy and Clinical Immunology [1] as a contribution to the Clinical Immunology Congress, 26-30 May 2018, Munich, Germany.

\section{References}

[1] T. I. Eliseeva, I. I. Balabolkin, E. S. Zastelo et al., "Blood counts in patients with acute hypersensitivity reactions to drugs and food," Allergy-European Journal of Allergy and Clinical Immunology, vol. 73, no. S105, p. 804, 2018.

[2] A. A. Justiz Vaillant and P. M. Zito, Immediate Hypersensitivity Reactions, StatPearls, Treasure Island, FL, USA, 2019.

[3] A. Reshma and A. K. Baranwal, "Child with allergies or allergic reactions," The Indian Journal of Pediatrics, vol. 85, no. 1, pp. 60-65, 2018.

[4] A. Muraro, R. F. Lemanske Jr., M. Castells et al., "Precision medicine in allergic disease-food allergy, drug allergy, and anaphylaxis-PRACTALL document of the European academy of allergy and clinical Immunology and the American academy of allergy, asthma and Immunology," Allergy, vol. 72, no. 7, pp. 1006-1021, 2017.

[5] T. I. Eliseeva and I. I. Balabolkin, "Drug allergic reactions: current views (review)," Sovremennye Tehnologii V Medicine, vol. 8, no. 1, pp. 159-172, 2016.
[6] T. Shiohara, Y. Mizukawa, and Y. Aoyama, "Monitoring the acute response in severe hypersensitivity reactions to drugs," Current Opinion in Allergy and Clinical Immunology, vol. 15, no. 4, pp. 294-299, 2015.

[7] M. Kimura, Y. Ito, M. Shimomura et al., "Cytokine profile after oral food challenge in infants with food protein-induced enterocolitis syndrome," Allergology International, vol. 66, no. 3, pp. 452-457, 2017.

[8] P. G. Fraenkel, "Anemia of inflammation," Medical Clinics of North America, vol. 101, no. 2, pp. 285-296, 2017.

[9] G. Jordakieva, M. Kundi, P. Lemell et al., "Sublingual house dust mite immunotherapy has no impact on decrease of circulating erythrocytes upon airway allergen challenge in allergic rhinitis," Scientific Reports, vol. 7, no. 1, p. 2555, 2017.

[10] G. Jordakieva, J. Wallmann, R. Schmutz et al., "Peripheral erythrocytes decrease upon specific respiratory challenge with grass pollen allergen in sensitized mice and in human subjects," PloS one, vol. 9, no. 1, Article ID e86701, 2014.

[11] S.-Y. Kim, M.-H. Kim, and Y.-J. Cho, "Different clinical features of anaphylaxis according to cause and risk factors for severe reactions," Allergology International, vol. 67, no. 1, pp. 96-102, 2018.

[12] N. Raison-Peyron, “Cutaneous adverse drug reactions” are not always drug-induced," European Journal of Dermatology, vol. 23, no. 4, pp. 439-442, 2013.

[13] G. Balakirski and H. F. Merk, "Cutaneous allergic drug reactions: update on pathophysiology, diagnostic procedures and differential diagnosic," Cutaneous and Ocular Toxicology, vol. 36, no. 4, pp. 307-316, 2017.

[14] W. Hoetzenecker, M. Nägeli, E. T. Mehra et al., "Adverse cutaneous drug eruptions: current understanding," Seminars in Immunopathology, vol. 38, no. 1, pp. 75-86, 2016.

[15] A. Elzagallaai, M. Greff, and M. Rieder, "Adverse drug reactions in children: the double-edged sword of therapeutics," Clinical Pharmacology \& Therapeutics, vol. 101, no. 6, pp. 725-735, 2017.

[16] M. R. McGill, "The past and present of serum aminotransferases and the future of liver injury biomarkers," EXCLI Journal, vol. 15, pp. 817-828, 2016.

[17] E. Adisen, M. A. Gürer, and Ö. Erdem, "Tetracycline/ doxycycline-induced cutaneous depressed pigmentation," International Journal of Dermatology, vol. 45, no. 10, pp. 1245-1247, 2006.

[18] J. Uetrecht and D. J. Naisbitt, "Idiosyncratic adverse drug reactions: current concepts," Pharmacological Reviews, vol. 65, no. 2, pp. 779-808, 2013.

[19] M. R. Ardern-Jones and P. S. Friedmann, "Skin manifestations of drug allergy," British Journal of Clinical Pharmacology, vol. 71, no. 5, pp. 672-683, 2011.

[20] W. J. Pichler, N. Yawalkar, M. Britschgi et al., "Cellular and molecular pathophysiology of cutaneous drug reactions," American Journal of Clinical Dermatology, vol. 3, no. 4, pp. 229-238, 2002.

[21] G. Garratty, "Immune hemolytic anemia caused by drugs," Expert Opinion on Drug Safety, vol. 11, no. 4, pp. 635-642, 2012.

[22] Q. A. Hill, R. Stamps, E. Massey et al., "Guidelines on the management of drug-induced immune and secondary autoimmune, haemolytic anaemia," British Journal of Haematology, vol. 177, no. 2, pp. 208-220, 2017.

[23] N. S. Young, "Acquired aplastic anemia," Annals of Internal Medicine, vol. 136, no. 7, pp. 534-546, 2002.

[24] O. Urbina, O. Ferrández, M. Espona, and S. Grau, "La importancia del síndrome de hipersensibilidad por 
antibióticos," Enfermedades Infecciosas y Microbiología Clínica, vol. 29, no. 7, pp. 558-559, 2011.

[25] C. Grigorakaki, F. Morceau, S. Chateauvieux, M. Dicato, and M. Diederich, "Tumor necrosis factor alpha-mediated inhibition of erythropoiesis involves GATA-1/GATA-2 balance impairment and PU.1 over-expression," Biochemical Pharmacology, vol. 82, no. 2, pp. 156-166, 2011.

[26] A. Brunyanszki, K. Erdelyi, B. Szczesny et al., "Upregulation and mitochondrial sequestration of hemoglobin occur in circulating leukocytes during critical illness, conferring a cytoprotective phenotype," Molecular Medicine, vol. 21, no. 1, pp. 666-675, 2015.

[27] J. Wu, Y. Y. Lee, S. C. Su et al., "Stevens-Johnson syndrome and toxic epidermal necrolysis in patients with malignancies," British Journal of Dermatology, vol. 173, no. 5, pp. 1224-1231, 2015.

[28] S.-H. Yun, E.-H. Sim, R.-Y. Goh, J.-I. Park, and J.-Y. Han, "Platelet activation: the mechanisms and potential biomarkers," BioMed Research International, vol. 2016, Article ID 9060143, 5 pages, 2016.

[29] P. Kolkhir, F. André, M. K. Church, M. Maurer, and M. Metz, "Potential blood biomarkers in chronic spontaneous urticaria," Clinical \& Experimental Allergy, vol. 47, no. 1, pp. 19-36, 2017.

[30] A. Kasperska-Zajac, A. Grzanka, J. Jarzab et al., "The association between platelet count and acute phase response in chronic spontaneous urticaria," BioMed Research International, vol. 2014, Article ID 650913, 6 pages, 2014.

[31] S. Singh, S. Khandpur, S. Arava et al., "Assessment of histopathological features of maculopapular viral exanthem and drug-induced exanthem," Journal of Cutaneous Pathology, vol. 44, no. 12, pp. 1038-1048, 2017. 